\title{
Investigation of curing rates of bio-based thiol-ene films from diallyl 2,5- furandicaboxylate
}

\author{
Larsen, Daniel Bo; Sønderbæk-Jørgensen, Rene; Duus, Jens Ø.; Daugaard, Anders E.
}

Published in:

European Polymer Journal

Link to article, DOI:

10.1016/j.eurpolymj.2018.03.005

Publication date:

2018

Document Version

Peer reviewed version

Link back to DTU Orbit

\section{Citation (APA):}

Larsen, D. B., Sønderbæk-Jørgensen, R., Duus, J. Ø., \& Daugaard, A. E. (2018). Investigation of curing rates of bio-based thiol-ene films from diallyl 2,5-furandicaboxylate. European Polymer Journal, 102, 1-8.

https://doi.org/10.1016/j.eurpolymj.2018.03.005

\section{General rights}

Copyright and moral rights for the publications made accessible in the public portal are retained by the authors and/or other copyright owners and it is a condition of accessing publications that users recognise and abide by the legal requirements associated with these rights.

- Users may download and print one copy of any publication from the public portal for the purpose of private study or research.

- You may not further distribute the material or use it for any profit-making activity or commercial gain

- You may freely distribute the URL identifying the publication in the public portal 


\section{Accepted Manuscript}

Investigation of curing rates of bio-based thiol-ene films from diallyl 2,5-furandicaboxylate

Daniel Bo Larsen, Rene Sønderbæk-Jørgensen, Jens Ø. Duus, Anders Egede Daugaard

PII: S0014-3057(17)31862-1

DOI: https://doi.org/10.1016/j.eurpolymj.2018.03.005

Reference: EPJ 8320

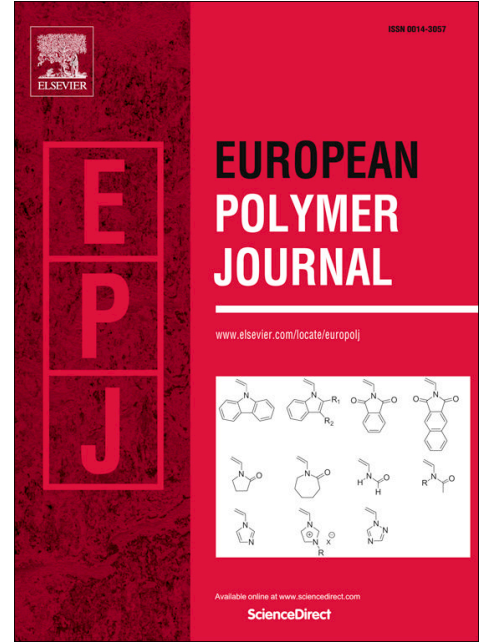

To appear in:

European Polymer Journal

Received Date:

10 November 2017

Revised Date:

8 February 2018

Accepted Date:

3 March 2018

Please cite this article as: Bo Larsen, D., Sønderbæk-Jørgensen, R., Duus, J.O., Egede Daugaard, A., Investigation of curing rates of bio-based thiol-ene films from diallyl 2,5-furandicaboxylate, European Polymer Journal (2018), doi: https://doi.org/10.1016/j.eurpolymj.2018.03.005

This is a PDF file of an unedited manuscript that has been accepted for publication. As a service to our customers we are providing this early version of the manuscript. The manuscript will undergo copyediting, typesetting, and review of the resulting proof before it is published in its final form. Please note that during the production process errors may be discovered which could affect the content, and all legal disclaimers that apply to the journal pertain. 


\section{Investigation of curing rates of bio-based thiol-ene films from diallyl 2,5-furandicaboxylate}

Daniel Bo Larsen ${ }^{1,2}$, Rene Sønderbæk-Jørgensen ${ }^{1}$, Jens $\emptyset$. Duus ${ }^{2}$, Anders Egede Daugaard $^{1}$

${ }^{1}$ Danish Polymer Centre (DPC), Department of Chemical and Biochemical Engineering, Technical University of Denmark, Søltofts Plads Building 227, 2800 Kgs. Lyngby, Denmark.

${ }^{2}$ Department of Chemistry, Technical University of Denmark, Kemitorvet, Bygning 207, $2800 \mathrm{Kgs}$. Lyngby, Denmark.

Correspondence to: Anders Egede Daugaard (E-mail: adt@kt.dtu.dk)

\section{Abstract}

The bio-based monomer, 2,5-furandicarboxylic acid, has been adapted to classic thiol-ene chemistry by derivatization of the acid with allyl alcohol. This new monomer has allowed for the synthesis of new thermoset systems, capable of forming green, sustainable materials through UV-crosslinking. In this study, the synthesis of the new monomer along with thorough kinetic studies of the new thermoset systems are presented. In order to determine kinetic values for the systems, all reactions have been followed by real-time FT-IR. Initially, a study of three different photoinitiators is performed on a classic TEMPIC-TATATO system, in order to determine the superior initiator for the new systems. The new monomer is crosslinked with five different thiol compounds in both stoichiometric and off-stoichiometric ratios, yielding an array of bio-based thermosets. The properties of these systems are determined through DSC, TGA and tensile testing, allowing determination of the systems with superior properties. In general, most systems proved to cure fully, with the exception of issues encountered from thiols with long ethoxylated chains.

\section{Introduction}

As the world progresses and oil reserves dwindle, the search for substitute resources intensifies [1] driven by increasing necessity. With an array of secondary products from bio-mass [2], this opens up for new solutions replacing traditional materials. For the polymer industry, the search has led to bio-mass derivatives capable of replacing their petro-based counterparts, either by drop-in bio-based monomers directly replacing materials such as ethylene, propylene and methacrylic acid [3], or brand new types of monomers such as methyl vinyl glycolate (MVG) [4] or trans-2,5-dihydroxy-3- 
pentenoic acid methyl ester (DPM) [5], leading to novel types of bio-based plastics. A monomer receiving interest is glycerol, which is obtained from hydrolysis of triglycerides found in plant and animal biomass [6] and can be used for block co-polymers [7], or as branch points in polyesters [8]. The bio-based derivative polylactic acid (PLA) has found use as an alternative to the petro-based polymers used in the packaging industry given its bio-degradability [9] as well as in nanocomposites with improved gas permeability[10,11]. 5-Hydroxymethylfurfural (HMF) has previously been identified as green monomer of interest due to its multiple applications as bio-based platform molecule [12]. It is obtained by dehydration without the use of metals from simple sugars and polysaccharides [13], and is seeing applications for bulk chemicals and bio-fuels, for example through oxidation to 2,5-furandicarboxylic acid (FDCA), a monomer proven suitable for synthesis of polyesters (Scheme 1). FDCA has previously been obtained through oxidation of HMF by ruthenium hydroxide [14], but has recently been produced through $\mathrm{Cu}$ catalyzed oxidation in good yields [15]. FDCA is a bio-based monomer, which has been receiving a great deal of interest, mainly due to its great resemblance to terephthalic acid (TPA), which, when polymerized with ethylene glycol, is used for the production of polyethylene terephthalate (PET) in the production of synthetic fibers and plastic bottles. The petro-based nature of TPA has led to a search for a suitable analogue and a probable solution has been the material polyethylene furanoate (PEF), a polyester of FDCA and ethylene glycol, which in many ways resemble PET and which is currently in use for this very purpose.[1] The potential of using PEF as replacement for PET plastics paves the way for a new market that is expected to reduce the price of FDCA over the coming years.

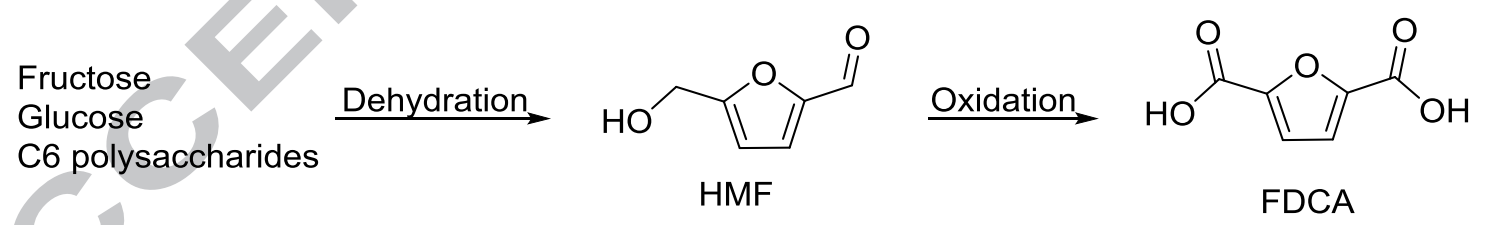

Scheme 1: Synthetic route for the production of $H M F$ and FDCA.

Many other bio-based building blocks have been developed over the last years. An example of this is allyl alcohol, which can be produced from catalytic conversion of glycerol through catalytic deoxydehydration reaction with transition metals such as rhenium [16,17].

In this paper, we report the derivatization of FDCA to the diallyl ester, diallyl furan-2,5dicarboxylate (DAFDC) with allyl alcohol, in order to provide a new polymer building block. This potential building block will be employed in thiol-ene chemistry with bio-based or partly bio-based thiol compounds, which through photo polymerization could yield a new thermoset material. Thiol- 
ene networks $[18,19]$ have previously been shown to have highly desirable mechanical behavior, due to narrow glass transition temperatures [20] and the uniformity and conversion of the crosslinking. Such behavior includes lower stress in films and higher adhesion to surfaces [21]. Building a cross-linked system through thiol-enes also gives the opportunity for off-stoichiometric systems [22] producing a functionalizable surface, which have been exploited for enzyme immobilization $[23,24]$ and particles $[25,26]$

We aim to produce several new types of thermoset systems, utilizing the new DAFDC building block in connection with different multifunctional thiol compound shown in Scheme 2. Most of the chosen thiol compounds contain some degree of bio-based components, increasing the overall green content of the targeted systems. This will expand the application of the bio-based FDCA to the classically known thiol-ene chemistry, yielding the opportunity for novel bio-based thermosets.
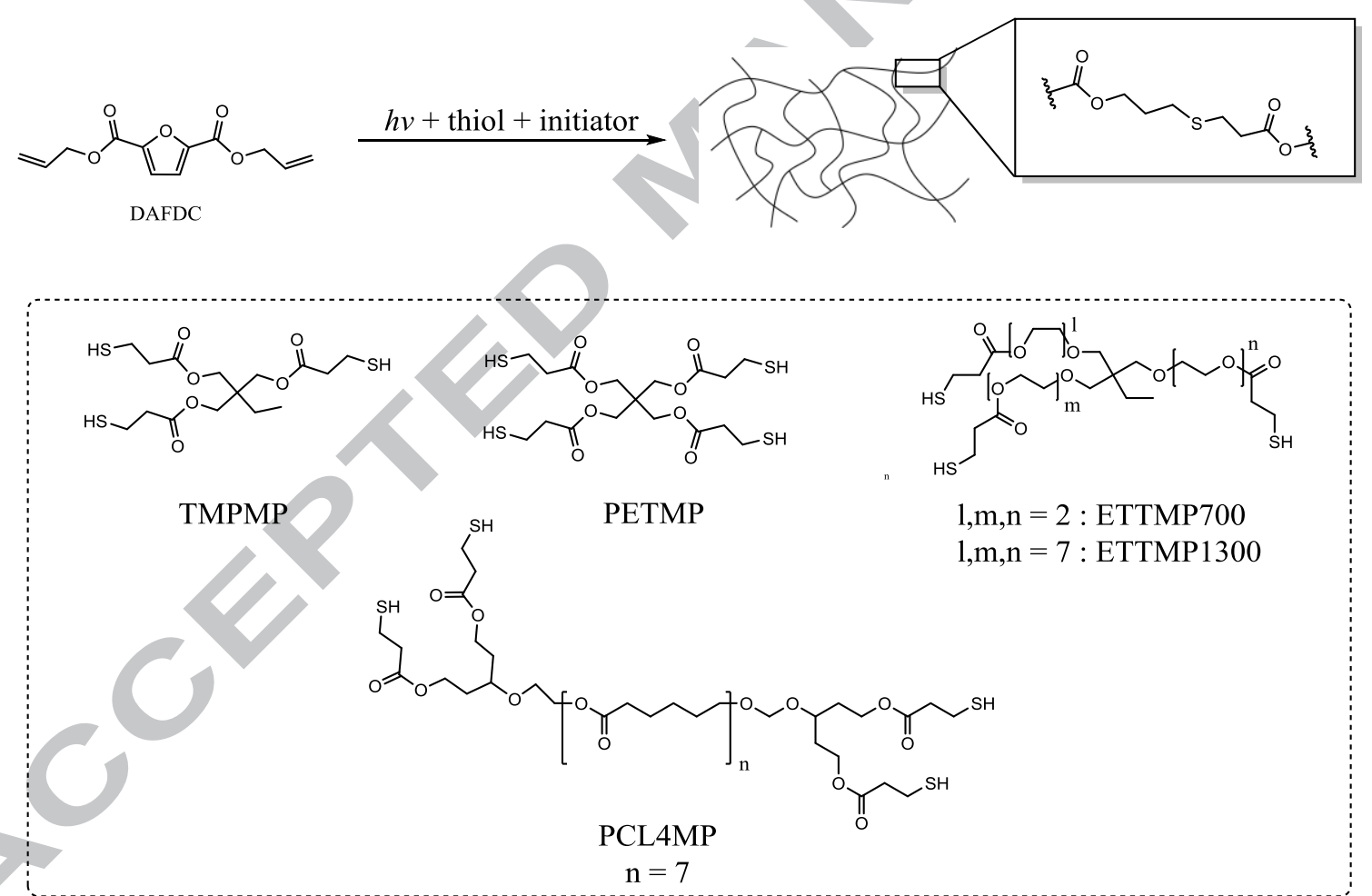

Scheme 2: Reaction scheme for the targeted thermoset systems and the various examined multifunctional thiols.

\section{Experimental}

\subsection{Chemicals}

The chemicals used were all purchased with the exception of DAFDC, which was produced in laboratories at DTU Department of Chemistry. Acetone (HPLC grade, 99.8 \%), 1,3,5-triallyl-1,3,5- 
triazine-2,4,6- $(1 \mathrm{H}, 3 \mathrm{H}, 5 \mathrm{H})$-trione $(98 \%)$, trimethylolpropan tri(3-mercaptopropionate) and pentaerythriol tetrakis(3-mercaptopropionate) (>95\%) were acquired from Sigma Aldrich® and used as received. Tris[2-(3-mercaptopropionyloxy)ethyl]isocyanurate, ethoxilatedtrimethylolpropan tri(3-mercaptopropionate) (THIOCURE® ETTMP 700), ethoxilatedtrimethylolpropan tri(3-mercaptopropionate) (THIOCURE® ETTMP 1300 ) and polycaprolactone tetra(3-mercaptopropionate) (THIOCURE® PCL4MP 1350) were obtained from Bruno Bock Thiocure ${ }^{\circledR}$ and used as received. The photo initiators Ommirad 1000 and Omnirad 4265 came from IGM Resins ${ }^{\circledR}$ while Lucirin is a product of BASF, all used as received. The three commercially available photo initiators used are Lucirin (P1) a yellow initiator consisting only of ethyl(2,4,6trimethylbenzoyl)-phenyl phosphinate, Ommirad 1000 (P2) a clear initiator consistsing of 80\% Omnirad 73 (2-hydroxy-2-methyl-1-phenylpropanone) and 20\% Omnirad 481 hydroxycyclohexyl-phenyl ketone) and Omnirad 4265 (P3) a slightly yellow initiator consisting of 50\% Lucirin and 50\% Omnirad 481.

\subsection{Method and characterization}

Identification of thiol-ene compounds and determination of reaction kinetics were performed on Nicolet iS50 FT-IR equipped with an iS50 ATR crystal from Thermo Scientific. The software used for the analysis was Omnic 9.7.46 firmware version 1.14 along with the macro software Macro Basic version 10.0.

The thiol-ene mixture was kept in darkness until the polymerization was initiated by a UV-LAMP $(365 \mathrm{~nm}, 11 \mathrm{~W}, 230 \mathrm{~V})$ as the only light source. A series of spectra were recorded with scans taken every four seconds until the reaction speed decreased and the ratio of scans was reduced to 24 seconds intervals and later 1 minute. The reaction was irradiated until no further reaction was detected (no decrease in the functional groups of interest). The allylic conversion was monitored through the allylic C-H out-of-plane bend at $930 \mathrm{~cm}^{-1}$ to $940 \mathrm{~cm}^{-1}$, as seen from Figure 1, and calculated using Equation (1) where $A(0)$ is the initial absorption and $A(t)$ is the adsorption at the time $t$. Reactions were monitored until no observable integral changes were present.

$$
x(t)=\frac{A(0)-A(t)}{A(0)} * 100 \%
$$

The maximum reaction rates were determined at the point with highest conversion rate $(\% / s)$ and the inhibition phase as the intercept of the tangent to this point with the $t$-axis. The maximum 
conversion was determined as the overall highest conversion observed. For estimation of total reaction time, the reaction was defined as complete when the reaction rate was less than $0.05 \% / s$.

Glass transition temperatures $\left(T_{g}\right)$ and melting temperatures $\left(T_{m}\right)$ were recorded using a TA instruments Discovery DSC. The thermal analysis was performed with a heating/cooling cycle of 10 ${ }^{\circ} \mathrm{C} / \mathrm{min}$ ranging from $-90{ }^{\circ} \mathrm{C}$ to $210{ }^{\circ} \mathrm{C}$. $T_{g}$ was measured at the inflection point and $T_{m}$ at the peak temperature. The thermogravimetric analysis (TGA) data supplied was obtained using TA instruments Discovery TGA. The measurement was performed from room temperature to $700{ }^{\circ} \mathrm{C}$.

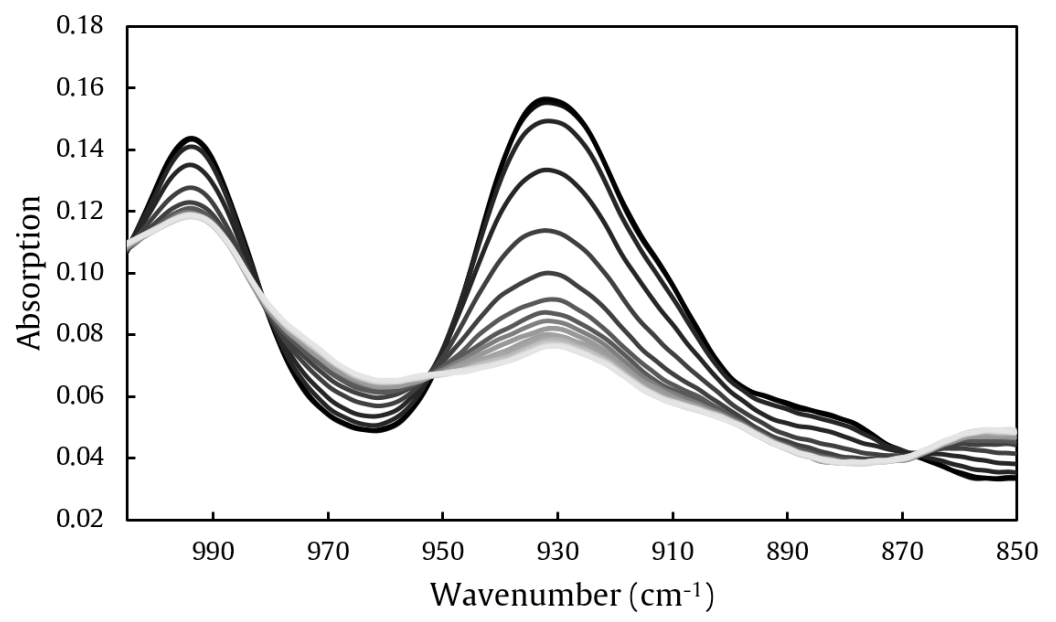

Figure 1: Real-time FTIR absroption spectra of the thiol-ene TEMPIC-TATATO system with the absorption $930 \mathrm{~cm}^{-1} \mathrm{corresponding}^{-}$ to the allylic $C$ - $H$ out-of-plane bend of DAFDC.

Nuclear Magnetic Resonance (NMR) spectra were obtained on a 400MHz Bruker Ascend magnet with an Avance II console and equipped with a Prodigy cryoprobe, at $400.13 \mathrm{MHz}$ for ${ }^{1} \mathrm{H}$ and 100.61 for ${ }^{13} \mathrm{C}$. Samples were dissolved in DMSO and the chemical shift reference set at 2.54 PPM. Data were processed and plotted using Mnova software.

Tensile tests were performed on a TA Electroforce equipped with a $45 \mathrm{~N}$ load cell. Tests were carried out on dog-bones, molded with a cut-out steel plate on a glass surface with the length, width and thickness of the dog-bone being $25.0 \mathrm{~mm}, 5.0 \mathrm{~mm}$ and $0.5 \mathrm{~mm}$ respectively. The dog-bones were pulled at a rate of $0.1 \mathrm{~mm} / \mathrm{s}$ for a total of $12 \mathrm{~mm}$ (the maximum of the instrument) or until rupture occurred. Young's moduli of the samples were determined from the slope of the initial 0.3 $\mathrm{mm}$ of the test, and ultimate strength and maximum strain were determined from the rupture point. All systems were tested with quintuplicate measurements. 


\subsection{Synthesis of diallyl furan-2,5-dicarboxylate}

2,5-furandicarboxylic acid (100 g, $0.513 \mathrm{~mol})$ was suspended in allyl alcohol (1000 mL) and concentrated sulfuric acid ( $2 \mathrm{~mL})$ was added. The suspension was heated to reflux for 72 hours, yielding a clear yellow solution. Following cooling to room temperature, the reaction mixture was concentrated to near dryness, yielding a black residue, which was dissolved in diethyl ether (1000 $\mathrm{mL})$, and washed twice with a saturated sodium bicarbonate solution $(2 \times 1000 \mathrm{~mL})$. The organic phase was concentrated yielding a yellow solid, which upon distillation (b.p. $140{ }^{\circ} \mathrm{C}$ at 15 mbar. Corrected b.p. $\left.276^{\circ} \mathrm{C}\right)$ yielded the desired diallyl furan-2,5-dicarboxylate $(92.2 \mathrm{~g}, 76 \%)$.

${ }^{1} \mathrm{H}$ NMR (400 MHz, DMSO- $\left.d_{6}\right) \delta 7.47$ (d, $\left.J=2.3 \mathrm{~Hz}, 1 \mathrm{H}\right), 6.02$ (ddt, $J=17.4,10.7,5.5 \mathrm{~Hz}, 1 \mathrm{H}$ ), $5.45-5.25(\mathrm{~m}, 2 \mathrm{H}), 4.82(\mathrm{dt}, J=5.6,1.4 \mathrm{~Hz}, 2 \mathrm{H})$.

${ }^{13} \mathrm{C}$ NMR $\left(101 \mathrm{MHz}, \mathrm{DMSO}-d_{6}\right) \delta 157.0,146.0,132.0,119.3,118.7,65.6$.

FTIR $\left(\mathrm{cm}^{-1}\right)$ : 470, 505, 562, 602, 622, 769, 815, 862, 924, 955.41, 982, 995, 1010, 1035, 1098, $1158,1231,1272,1293,1377,1386,1417,1454,1507,1572,1653,1717,3013,3110$

\subsection{General procedure: Synthesis of thiol-ene TEMPIC/TATATO thin film}

The general procedure applies to all the experiments performed for this paper exemplified with this synthesis (see supporting information for all thiol-ene procedures). The thiol component Tris[2-(3mercaptopropionyloxy)ethyl]isocyanurate (TEMPIC) (1.393 g, $2.65 \mathrm{mmol})$ was added to the allylic component 1,3,5-triallyl-1,3,5-triazine-2,4,6-(1H,3H,5H)-trione (TATATO) (0.672 g, 2.69 mmol) and the initiator Lucirin $(5.04 \mathrm{mg}, 0.016 \mathrm{mmol})$. The mixture was dissolved in acetone $(1 \mathrm{ml})$ and stirred on a vortex mixer at $2500 \mathrm{rpm}$ for approximately one minute or until fully dissolved. While being kept in darkness two drops of the solution were added to the ATR crystal with a pipette yielding a film of approximately $1 \mathrm{~mm}$ in thickness. Ambient light was blocked so that only the light from the UV-lamp initiated the reaction.

\subsection{General procedure: Synthesis of films for tensile testing}

Diallyl furan-2,5-dicarboxylate (2.20 g, $9.29 \mathrm{mmol})$, Pentaerythritol tetrakis(3-mercaptopropionate) $(2.40 \mathrm{~g}, 4.66 \mathrm{mmol})$ and OMNIRAD 4265 (109 mg) were weighed in a cup and mixed on a speed mixer for 10 minutes at $3500 \mathrm{RPM}$ or until homogenous. The mixture was poured into a cut-out steel plate with dog-bone-shapes mounted on a glass plate and any excess was removed with an industrial razorblade. The plate was placed in an UV-chamber $(365 \mathrm{~nm})$ for 10 minutes yielding the cured dog-bones for tensile testing. 


\section{Results and discussion}

\subsection{Monomer synthesis}

We synthesized DAFDC through acidic esterification with allyl alcohol and a catalytic amount of sulfuric acid (Scheme 3).

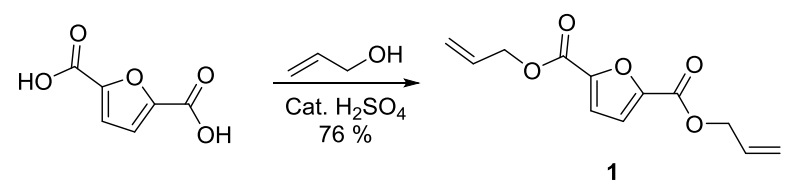

Scheme 3: Synthesis of the DAFDC monomer.

This monomer has been synthesized in similar fashion previously [27], and was originally reported as a yellow oil. Initially we observed the same appearance, with both IR and NMR experiments showing apparent sufficient purity for further experiments. However, it was proven that small amounts of impurities were present in the yellow oil, as evidenced by the fact that high temperature experiments lead to degradation to a black residue. This could be circumvented by subjecting the compound to a basic wash followed by a distillation, yielding the monomer as a white crystalline solid. This pure material could be used in high temperature reactions without the formation of black residue. Since direct distillation of the yellow oil without basic wash yielded the same black residue, it was concluded that the impurities stem from minor traces of acid. NMR spectroscopy of the black residue revealed relative increase in the amount of aromatic protons (Figure 2), leading to the theory that the degradation of the compound could derive from a possible inverse electron demand Diels-Alder reaction between the allyl groups and the furan ring. This creates an oxygenbridged six membered ring system, which, through dehydration, yields aromatic benzene rings (Scheme 4).

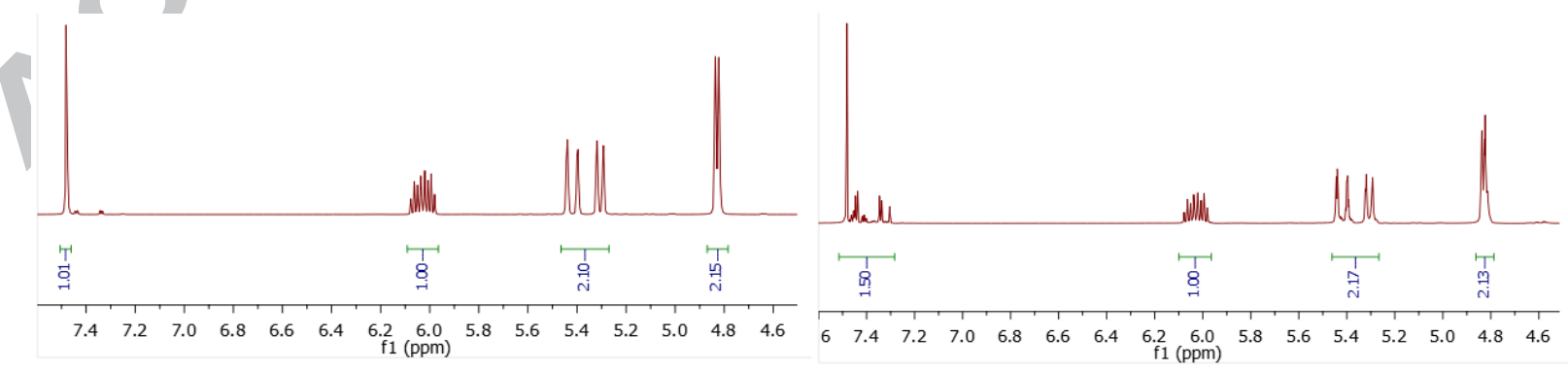

Figure 2: H-NMR spectra of the DAFDC monomer (left) and the obtained black residue from heating (right) showing increased integral for the aromatic region at $\delta \approx 7.4 \mathrm{ppm}$. 


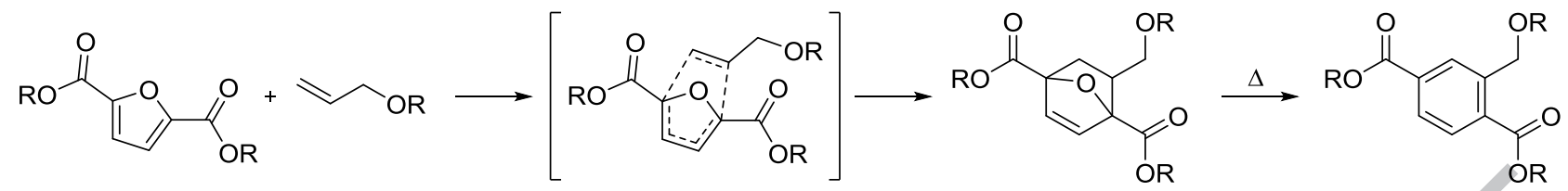
Scheme 4: Proposed mechanism for Diels-Alders reaction and following dehydration of the furane ring and the allyl group at high temperatures.

\subsection{Photoinitiators and kinetics}

In order to evaluate the strengths and drawbacks of different photoinitiator systems we tested three commercially available photoinitiators, Lucrin (P1), Ommirad 1000 (P2) and Omnirad 4265 (P3), in the classically known thiol-ene reaction of TEMPIC and TATATO, by using $0.25 \mathrm{wt} \%, 0.05 \mathrm{wt} \%$ and $0.025 \mathrm{wt} \%$ of the initiators, respectively. The conversion was calculated using Equation (1) and the results are presented in Figure 3 with accommodating data in Table 1.

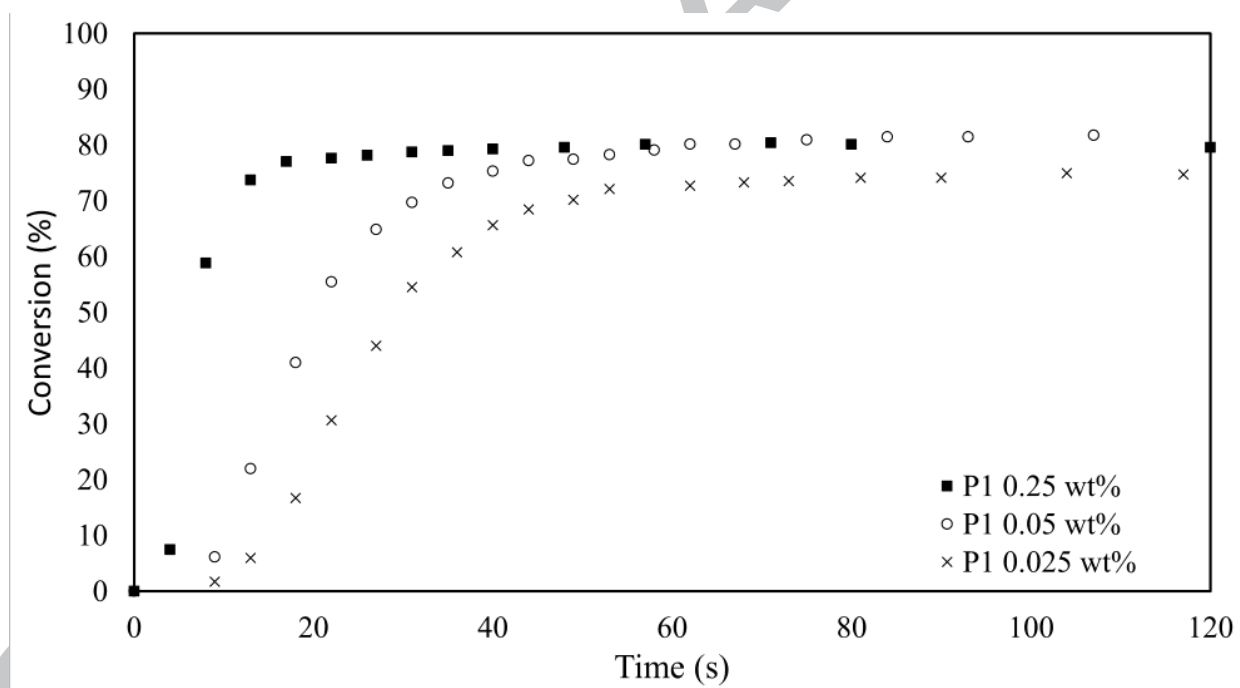



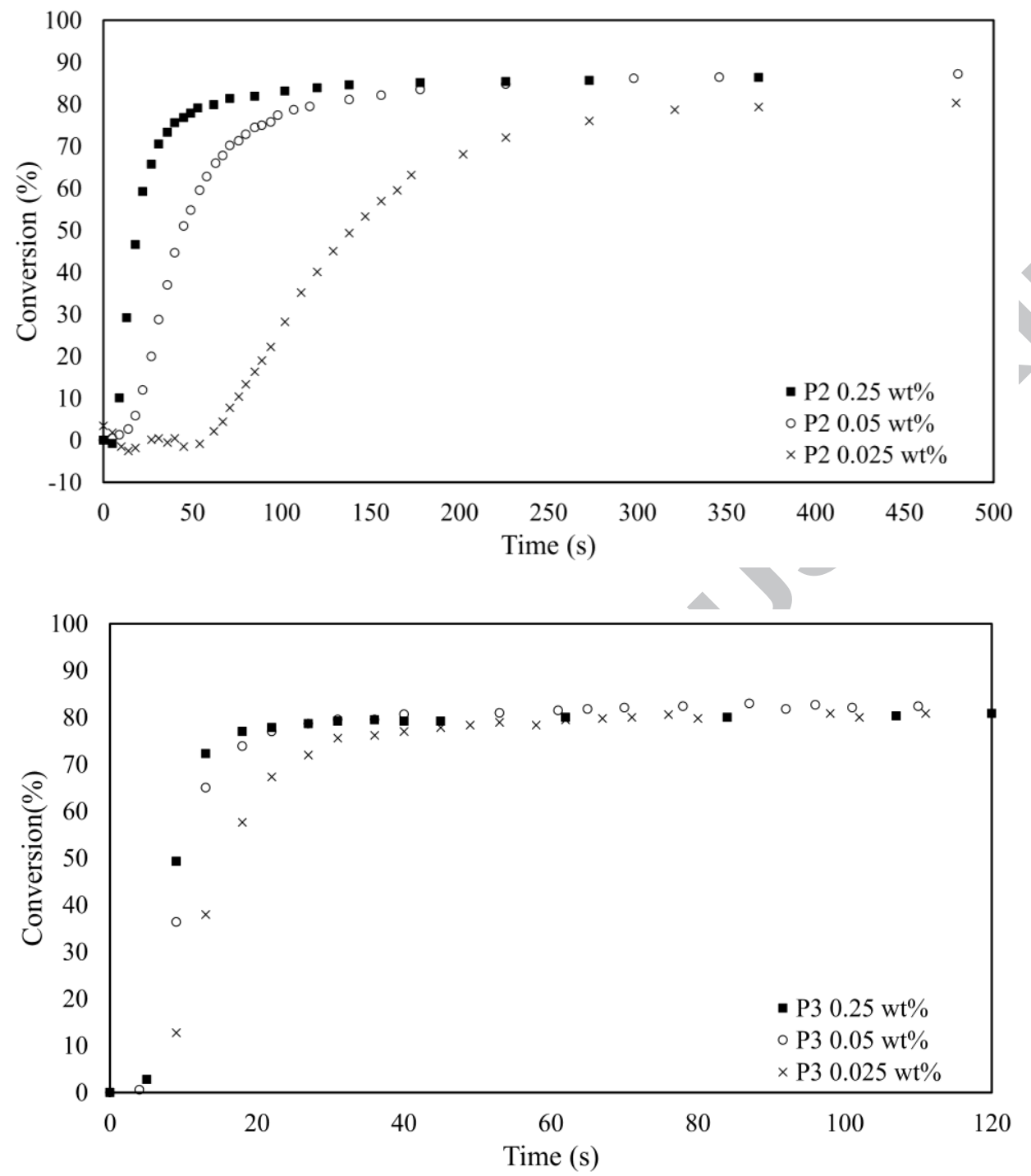

Figure 3: Allylic conversion of the TEMPIC-TATATO system with the three different examined photoinitiators.

\begin{tabular}{lccccc}
\hline Sample & Max. Rate $(\% / \mathrm{s})$ & Rel. Rate & Inh. Phase $(\mathrm{s})$ & Max. Conv. $(\%)$ & Conv. Time (s) \\
\hline P1 $0.25 \mathrm{wt} \%$ & 12.85 & 1 & 3.4 & 82.04 & 48 \\
P1 $0.05 \mathrm{wt} \%$ & 3.95 & 0.31 & 7.4 & 85.79 & 107 \\
P1 $0.025 \mathrm{wt} \%$ & 3.48 & 0.27 & 13.2 & 76.99 & 117 \\
P2 $0.25 \mathrm{wt} \%$ & 4.79 & 0.37 & 6.9 & 87.66 & 85 \\
P2 $0.05 \mathrm{wt} \%$ & 2.19 & 0.17 & 17.9 & 88.56 & 226 \\
P2 $0.025 \mathrm{wt} \%$ & 0.83 & 0.06 & 65.8 & 78.22 & 368 \\
P3 0.25 wt\% & 11.63 & 0.91 & 4.8 & 82.83 & 40 \\
P3 0.05 wt\% & 7.17 & 0.56 & 3.9 & 84.38 & 136 \\
P3 0.025 wt\% & 6.30 & 0.49 & 7.0 & 82.55 & 151 \\
\hline
\end{tabular}

Table 1: Kinetic data for the TEMPIC-TATATO system with the different photoinitiators. 
A general trend observed is that the amount of initiator is limiting for the reaction rate. Most reactions exhibited short inhibition phases, with the exception of $\mathrm{P} 2$, which shows longer inhibition times present in less than $0.25 \mathrm{wt} \%$. In addition, the rate of reactions initiated by $\mathrm{P} 2$ are much lower compared to P1 and P3. The reactions of TEMPIC and TATATO show that the choice of initiator can limit the maximum conversion. All initiators show the highest conversion rates with a $0.05 \mathrm{wt} \%$ loading. For P1 and P2 it is observed that using only $0.025 \mathrm{wt} \%$ initiator limits the maximum conversion by $5-10 \%$, whereas the conversion rates when utilizing P3 remain predominantly constant. P3 has thereby shown to have superior kinetic properties under the given conditions in the acetone diluted thiol-ene composition. Meanwhile the conversion percentage does not seem to be affected by the three initiators themselves, which has led to P3 being the chosen initiator for further testing. A factor contributing to the higher performance of the P1 and P3 systems compared to P2, is most likely the choice of the UV source with a $365 \mathrm{~nm}$ wavelength, since P1 and P3 have absorption wavelengths at 370 and $379 \mathrm{~nm}$ respectively, whereas the highest absorption wavelength of $\mathrm{P} 2$ is $340 \mathrm{~nm}$.

\subsection{Synthesis of bio-thiol-ene thin films}

From the initial studies of the photoinitiators, P3 was deemed the most efficient. With this, the targeted bio-thiol-ene cross-linked networks were polymerized using equimolar amount of functional groups i.e. allyl and thiol groups and $2.5 \mathrm{wt} \%$ P3 initiator for all five thiol monomers shown in Scheme 2. The amount of initiator has been increased due to the slower propagation rate of of DAFDC compared to TATATO in the thiol-ene system, and the ten-fold increase of initiator was required in order to obtain reaction rates equivalent to those observed for the TEMPIC/TATATO system. The conversions were calculated as before using equation (1) with the resulting curves presented in Figure 4 (conversion curves for off-stoichiometric systems are presented in Supporting Information, Figure S2 and S3). 


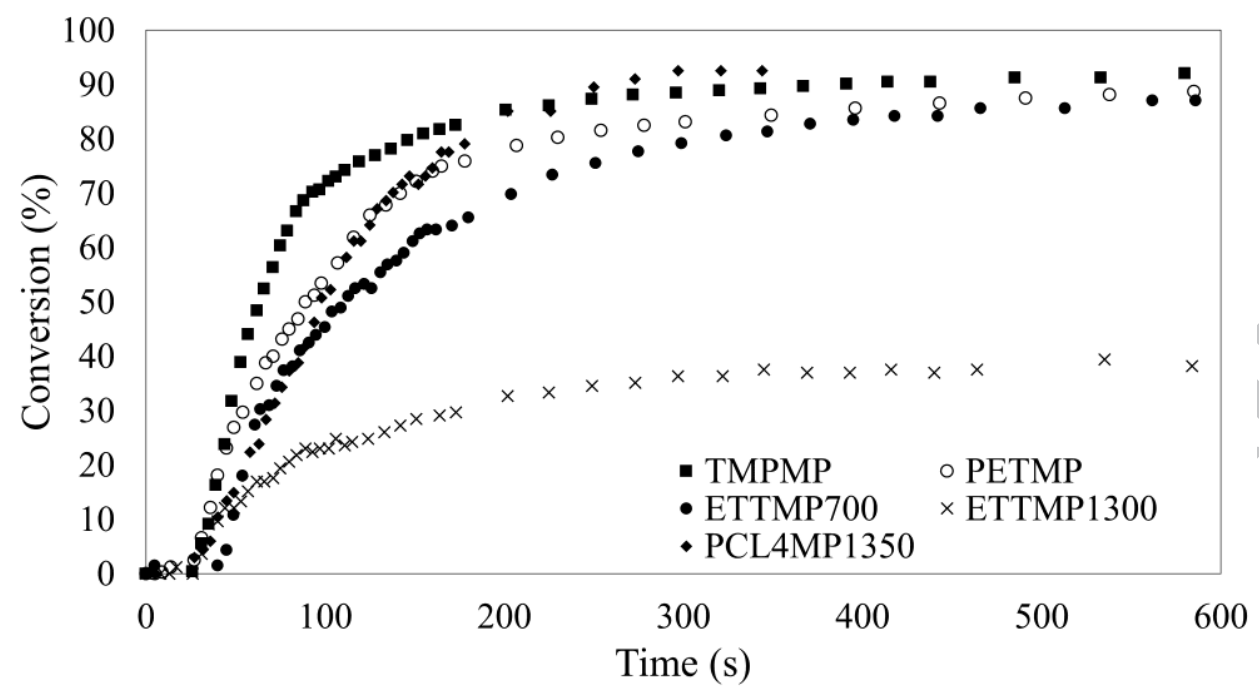

Figure 4: Allylic conversion of 1:1 SH/C=C stoichiometric systems with DAFDC, the different thiols and 2.5 wt\% P3.

The five different thiol monomers represents a variety of different functionalities and are the standard for a thiol-ene system. To examine how the system reacted to off-stoichiometric ratios, each of the systems was polymerized using both a 1.3 stoichiometric surplus of allyl and thiol groups. Using Carothers equation and the statistical method the theoretical gel-point has been calculated for tri- and tetra functional thiol compounds with a di-functional allyl compound before experiments started. Calculated critical conversions are given in Table S1 and S2 (see Supporting Information). The thiol-ene reactions using DAFDC as the allylic monomer all yielded cross-linked products each with different properties. Most of the thiol-ene systems reached high levels of conversion and yielded solid samples with varying mechanical properties. ETTMP1300 based systems did not fully cure, but yielded coherent flexible films with sticky surfaces, which were observed to be unstable over time (both ETTMP700 and ETTMP1300). The systems reverted to a viscous liquid state after storage for several months, with IR spectra showing a large broad peak at $\sim 3400 \mathrm{~cm}^{-1}$ consistent with formation of hydroxyl groups, indicating hydrolysis of the PEG chains (Supporting Information, Figure S4).

Conversion rates were measured with FT-IR as for the TEMPIC-TATATO system, and all kinetic data are shown in Table 2. 


\begin{tabular}{lcccccc}
\hline Thiol & {$[\mathrm{SH}]:[\mathrm{C}=\mathrm{C}]$} & Max. Rate $(\% / \mathrm{s})$ & Rel. Rate & Inh. Phase $(\mathrm{s})$ & Max. Conv. $(\%)$ & Conv. Time $(\mathrm{s})$ \\
\hline TMPMP & $1.0: 1.0$ & 2.0 & 1.00 & 32.0 & 92.9 & 225 \\
TMPMP & $1.3: 1.0$ & 1.8 & 0.89 & 13.4 & 92.6 & 296 \\
TMPMP & $1.0: 1.3$ & 1.2 & 0.61 & 22.3 & 84.8 & 178 \\
PETMP & $1.0: 1.0$ & 1.5 & 0.75 & 27.8 & 91.6 & 278 \\
PETMP & $1.3: 1.0$ & 2.4 & 1.20 & 12.8 & 91.5 & 207 \\
PETMP & $1.0: 1.3$ & 2.7 & 1.38 & 6.0 & 84.5 & 202 \\
ETTMP700 & $1.0: 1.0$ & 1.6 & 0.82 & 42.3 & 89.2 & 513 \\
ETTMP700 & $1.3: 1.0$ & 3.1 & 1.54 & 8.8 & 88.6 & 202 \\
ETTMP700 & $1.0: 1.3$ & 1.1 & 0.54 & 20.5 & 77.0 & 298 \\
ETTMP1300 & $1.0: 1.0$ & 1.4 & 0.69 & 28.3 & 39.4 & 369 \\
ETTMP1300 & $1.3: 1.0$ & 0.9 & 0.47 & 11.6 & 32.5 & 249 \\
ETTMP1300 & $1.0: 1.3$ & 0.9 & 0.45 & 78.0 & 45.2 & 250 \\
PCL4MP1350 & $1.0: 1.0$ & 1.1 & 0.57 & 24.3 & 92.5 & 321 \\
PCL4MP1350 & $1.3: 1.0$ & 4.7 & 2.39 & 9.6 & 94.8 & 71 \\
PCL4MP1350 & $1.0: 1.3$ & 1.7 & 0.86 & 27.5 & 83.8 & 298 \\
\hline & & Table 2: Kinetic data for the synthesized thermoset systems. &
\end{tabular}

The conversion speeds of the thiol-ene reactions (Table 2) were greatly affected by the choice of thiol monomer. High reaction rates are observed when employing the PCL4MP1350 thiol in surplus. While the ETTMP monomers exhibit generally lower reaction rates and inferior conversions, especially ETTMP1300. This phenomena is attributed to the aforementioned instability of the systems, which was observable within the timeframe of the experiments for ETTMP1300 (Supporting Information, Figure S1). This also show that the higher molecular weight ETTMP monomers are slower reacting and more unstable compared to their lower molecular weight counterparts. ETTMP1300 has long ethoxylated chains, which likely prevents complete curing, supported by the conversions between 30 and $45 \%$ and lower conversions obtained with increased amounts of the thiol component.

The thiol-enes are fully capable of reacting off-stoichiometrically while maintaining approximately the same conversion, around $90 \%$. Naturally, due to conversion being measured from the IR signal of allyl groups, systems with an excess of allyl functionality exhibit lower conversions. Though with conversions above $77 \%$ (the natural stoichiometric limitation), it is suspected that some allylallyl propagation is occurring besides the expected thiol-allyl crosslinks. The even distribution in maximum conversion can be contributed to restrictions of the network preventing further reaction once it has reached the gel-point. Comparing the DAFDC systems and a conventional system like TEMPIC-TATATO, a higher conversion of the functional groups are attained with the exception of ETTMP1300. 
All systems were subjected to DSC and TGA analysis, with their thermal properties being presented in Table 3. The data obtained by DSC shows a large variation of the thiol-ene systems with $T_{g}$ varying from $-61.4{ }^{\circ} \mathrm{C}$ to $9.3{ }^{\circ} \mathrm{C}$. In general, $T_{g}$ is heavily influenced by molecular weight and the rotational freedom of the compound, which can then be used as an indicator for the ability of the system to create networks. This is exemplified by PETMP systems having the highest $T_{g}\left(9.3{ }^{\circ} \mathrm{C}\right)$ of the five systems, resulting from its small size and high functionality. An absence of melting points was observed during the second heating cycle of the DSC scans. We expect this phenomenon to occur from slow crystallization of the polymers, hindering recrystallization of the systems. The TGA results (Table 3) generally show a small initial weight loss around $100^{\circ} \mathrm{C}$ corresponding to between 4 and $15 \%$, which is attributed to residual solvent trapped during the curing reaction. The primary degradation starts around $270{ }^{\circ} \mathrm{C}$ to $300{ }^{\circ} \mathrm{C}$ and ends at approximately at $400{ }^{\circ} \mathrm{C}$ to $500{ }^{\circ} \mathrm{C}$, losing approximately $90 \%$ of the total mass. It is clear that the content of DAFDC increases the thermal stability as well as the residual mass after degradation, where e.g. the PETMP systems (that are high in DAFDC) show the highest residue with $23 \%$ and the highest end temperature $\left(\mathrm{T}_{\text {end }}\right)$ of degradation. The overall order of performance of the thiol monomers based on the end of degradation are rated as PETMP > TMPMP > PCL4MP1350 > ETTMP700 > ETTMP1300 (Table 3). This order also reflects the instability of the ETTMP based systems. Regarding the onset of degradation, the performance of the systems change. Here the ETTMP1300 and PCL4MP1350 systems perform well, with degradations starting in the $285-300{ }^{\circ} \mathrm{C}$ interval, followed by PETMP, ETTMP700 and TMPMP respectively decreasing in stability. A general trend that is observed, is the stoichiometric systems tend to initiate degradation later than the off-stoichiometric systems, indicating an excess of functional groups contributing to the degradation process. 


\begin{tabular}{lcccccc}
\hline Thiol & {$[\mathrm{SH}]:[\mathrm{C}=\mathrm{C}]$} & $\operatorname{Tg}\left({ }^{\circ} \mathrm{C}\right)^{1}$ & $\operatorname{Tm}\left({ }^{\circ} \mathrm{C}\right)^{1}$ & $\mathrm{~T}_{\text {start }}$ degradation $\left({ }^{\circ} \mathrm{C}\right)$ & $\mathrm{T}_{\text {end }}$ degradation $\left({ }^{\circ} \mathrm{C}\right)$ & Mass loss $(\%)$ \\
\hline TMPMP & $1.0: 1.0$ & -8.5 & - & 267.8 & 521.5 & 89.9 \\
TMPMP & $1.3: 1.0$ & -54.8 & 135.0 & 268.4 & 521.4 & 83.0 \\
TMPMP & $1.0: 1.3$ & -24.5 & 137 & 271.6 & 521.4 & 87.3 \\
PETMP & $1.0: 1.0$ & 9.3 & 135 & 280.7 & 539.7 & 77.5 \\
PETMP & $1.3: 1.0$ & 9.19 & 124 & 278.3 & 547.0 & 73.4 \\
PETMP & $1.0: 1.3$ & 0.0 & 135 & 277.3 & 543.1 & 76.3 \\
ETTMP700 & $1.0: 1.0$ & -55.5 & 148 & 280.2 & 485.3 & 88.9 \\
ETTMP700 & $1.3: 1.0$ & -35.0 & - & 267.6 & 489.3 & 94.4 \\
ETTMP700 & $1.0: 1.3$ & -55.5 & 148 & 279.7 & 483.6 & 88.3 \\
ETTMP1300 & $1.0: 1.0$ & -57.0 & 162 & 301.4 & 464.3 & 93.0 \\
ETTMP1300 & $1.3: 1.0$ & -53.4 & - & 283.9 & 457.4 & 93.3 \\
ETTMP1300 & $1.0: 1.3$ & -49.5 & 162 & 281.0 & 466.4 & 91.0 \\
PCL4MP1350 & $1.0: 1.0$ & -36.0 & 190 & 299.3 & 508.0 & 90.8 \\
PCL4MP1350 & $1.3: 1.0$ & -61.4 & 160.0 & 286.4 & 518.8 & 91.6 \\
PCL4MP1350 & $1.0: 1.3$ & -55.0 & 150 & 291.6 & 511.1 & 88.7 \\
\hline${ }^{1}$ Deter & & &
\end{tabular}

${ }^{1}$ Determined from first DSC heating cycle

Table 3: Thermal properties of the synthesized thermoset systems.

To evaluate the mechanical properties of the examined bio-films, tensile tests were performed on a selection of the films. In order to observe both the effect of the various thiols and stoichiometric change in the reaction feed, all stoichiometric systems with the five different thiols were tested along with a test series of the PETMP system with the variances on stoichiometry. All results are summarized in Table 4 (plots of the tensile tests are presented in Supporting Information). The tests showed that off-stoichiometric ratios caused softer and more flexible materials, as anticipated due to a less densely crosslinked network. However, excess of thiols led only to a minor reduction in modulus, while an increase in tensile strength was observed. Contrary to this, an increase in allyl functionality led to a reduction of modulus by approximately $50 \%$ along with a lowering of tensile strength. This difference was attributed to the difference in functionality between the thiols and DAFDC, which effectively gives a better integrity in the network at higher amounts of the multifunctional thiols compared to excess of the bifunctional DAFDC.

The various thiols also resulted in great differences in mechanical behavior of the systems. PETMP, TMPMP based systems resulted in stiffer and stronger materials, while PCL4MP was stiff but of 
intermediate tensile strength and ETTMP700 and 1300 systems were much softer and exhibited almost elastomeric properties.

\begin{tabular}{lcccc}
\hline Thiol & {$[\mathrm{SH}]:[\mathrm{C}=\mathrm{C}]$} & Young's Modulus $(\mathrm{MPa})$ & Tensile Strength $(\mathrm{MPa})$ & Maximum Strain \\
\hline PETMP & $1.0: 1.0$ & $6.1 \pm 0.4$ & $1.1 \pm 0.3$ & $0.23 \pm 0.06$ \\
PETMP & $1.3: 1.0$ & $5.4 \pm 0.7$ & $1.2 \pm 0.1$ & $0.30 \pm 0.04$ \\
PETMP & $1.0: 1.3$ & $3.1 \pm 0.3$ & $0.75 \pm 0.09$ & $0.35 \pm 0.01$ \\
TMPMP & $1.0: 1.0$ & $3.0 \pm 0.3$ & $0.83 \pm 0.09$ & $0.38 \pm 0.04$ \\
ETTMP700 & $1.0: 1.0$ & $1.69 \pm 0.03$ & $0.44 \pm 0.09$ & $0.35 \pm 0.07$ \\
ETTMP1300 & $1.0: 1.0$ & $0.67 \pm 0.03$ & $\mathrm{~N} / \mathrm{A}$ & $\mathrm{N} / \mathrm{A}$ \\
PCL4MP1350 & $1.0: 1.0$ & $4.8 \pm 0.5$ & $0.5 \pm 0.1$ & $0.12 \pm 0.04$ \\
\hline
\end{tabular}

Table 4: Mechanical properties obtained from tensile testing.

In order to claim the thiol-ene systems as bio-based the thiol compounds have been scrutinized by retrosynthetic analysis. The core of TMPMP, ETTMP700 and ETTMP1300 is 2-ethyl-2(hydroxymethyl)propane-1,3-diol, 2,2-bis(hydroxymethyl)propane-1,3-diol for PETMP, which can be synthesized from propan-1-ol, n-butanol and formic acid that are all available from renewable resources [28][29]. The spacers used in ETTMP monomers are ethylene oxide which can be produced by oxidation of bio-ethylene [30]. 3-mercaptopropionate, the thiol constituent in all the thiol compounds, can be synthesized by hydrogen sulfide and but-3-enioc acid, which can be found as derivatives of bio-mass [31][32]. This makes thiol-ene systems based on TMPMP, PETMP and ETTMPs at least theoretically available as $100 \%$ bio-based systems. On the other hand, PCL4MP1350s main constituency is poly(caprolactone), which is still only a derivative of crude oil, resulting in a thiol-ene network such as T-PCL1 being $42 \mathrm{wt} \%$ bio-based.

\section{Conclusion}

In this study, we have presented a green synthetic route to the monomer DAFDC with high purity, using a simple procedure allowing effective upscaling. We have shown the necessity of thorough purification by distillation of this monomer in order to avoid unwanted side-reaction at higher temperatures. With the aim of using DAFDC in UV-initiated thiol-ene crosslinking reaction, we have studied the reaction rates of three different photo initiators in a model TEMPIC/TATATO system with the objective of determining the most suitable initiator. The results revealed Omnirad 4265 to be superior to both Lucirin and Omnirad 1000 under the tested conditions. Omnirad 4265 was then employed in the screening of DAFDC with five fully or partly bio-based thiol compounds, TMPMP, PETMP, ETTMP700, ETTMP1300 and PCL4MP. All five thiol compounds were shown to be fully capable of forming crosslinked networks in a thiol-ene reaction, and be able to maintain fast curing rates with the photo initiator. Generally, high conversions were observed for the curing 
and the reactions yielded materials with desirable thermal qualities, their glass transition temperatures ranging between $-61.4{ }^{\circ} \mathrm{C}$ and up to $9.3{ }^{\circ} \mathrm{C}$. Only ETTMP1300 systems showed conversion percentages too low for fully cross-linked networks and also displayed susceptibility to hydrolysis of the ethoxylated chains during storage. Mechanical properties of the systems were directly correlated to the type of thiol and stoichiometry of the mixture, where PETMP and TMPMP resulted in stiffer materials and highest tensile strength, PCL4MP resulted in an intermediate strength and ETTMP700 and ETTMP1300 systems were softer and weaker systems. With this study, we step towards the incorporation of the bio-based FDCA into known sulfide chemistry, giving the opportunity of numerous new sustainable thermoset systems. In future work, we aim at investigating the potential of such systems in composites or elastomers by use of reinforcing agents.

\section{Acknowledgements}

We would like to thank Peter Fristrup for initializing the project collaboration and Liyun Yu for help with thermal analysis as well as Bruno Bock and IGM Resins for supplying chemicals.

\section{References}

[1] A. Gandini, Monomers and Macromonomers from Renewable Resources, in: K. Loos (Ed.), Biocatal. Polym. Chem., Wiley-VCH Verlag GmbH \& Co. KGaA, 2010: pp. 1-33.

[2] S.N. Naik, V. V. Goud, P.K. Rout, A.K. Dalai, Production of first and second generation biofuels: A comprehensive review, Renew. Sustain. Energy Rev. 14 (2010) 578-597.

[3] L. Axelsson, M. Franzén, M. Ostwald, G. Berndes, G. Lakshmi, N.H. Ravindranath, Perspective: Jatropha cultivation in southern India: Assessing farmers' experiences, Biofuels, Bioprod. Biorefining. 6 (2012) 246-256.

[4] M. Dusselier, P. Van Wouwe, S. De Smet, R. De Clercq, L. Verbelen, P. Van Puyvelde, F.E. Du Prez, B.F. Sels, Toward functional polyester building blocks from renewable glycolaldehyde with sn cascade catalysis, ACS Catal. 3 (2013) 1786-1800.

[5] S.G. Elliot, C. Andersen, S. Tolborg, S. Meier, I. Sádaba, a. E. Daugaard, E. Taarning, Synthesis of a novel polyester building block from pentoses by tin-containing silicates, RSC Adv. 7 (2017) 985-996.

[6] H. Zhang, M.W. Grinstaff, Recent Advances in Glycerol Polymers: Chemistry and Biomedical Applications, Macromol. Rapid Commun. 35 (2014) 1906-1924.

[7] G.Z. Papageorgiou, V. Tsanaktsis, D.N. Bikiaris, Synthesis of poly(ethylene furandicarboxylate) polyester using monomers derived from renewable resources: thermal behavior comparison with PET and PEN, Phys. Chem. Chem. Phys. 16 (2014) 7946-7958.

[8] H. Nguyen, D. Löf, S. Hvilsted, A. Daugaard, Highly Branched Bio-Based Unsaturated Polyesters by Enzymatic Polymerization, Polymers (Basel). 8 (2016) 363-374. 
[9] H. Tsuji, Poly ( Lactic Acid ), in: Bio-Based Plast. Mater. Appl., John Wiley \& Sons Ltd, Chichester, UK, 2014: pp. 171-239.

[10] J. Trifol, D. Plackett, C. Sillard, P. Szabo, J. Bras, A.E. Daugaard, Hybrid poly(lactic acid)/nanocellulose/nanoclay composites with synergistically enhanced barrier properties and improved thermomechanical resistance, Polym. Int. 65 (2016) 988-995.

[11] J. Trifol, D. Plackett, C. Sillard, O. Hassager, A.E. Daugaard, J. Bras, P. Szabo, A comparison of partially acetylated nanocellulose, nanocrystalline cellulose, and nanoclay as fillers for highperformance polylactide nanocomposites, J. Appl. Polym. Sci. 133 (2016) 43257-43268.

[12] A.A. Rosatella, S.P. Simeonov, R.F.M. Frade, C. a. M. Afonso, 5-Hydroxymethylfurfural (HMF) as a building block platform: Biological properties, synthesis and synthetic applications, Green Chem. 13 (2011) 754-793.

[13] T. Ståhlberg, S. Rodriguez-Rodriguez, P. Fristrup, A. Riisager, Metal-Free Dehydration of Glucose to 5-(Hydroxymethyl)furfural in Ionic Liquids with Boric Acid as a Promoter, Chem. A Eur. J. 17 (2011) 1456-1464.

[14] Y.Y. Gorbanev, S. Kegnæs, A. Riisager, Selective aerobic oxidation of 5hydroxymethylfurfural in water over solid ruthenium hydroxide catalysts with magnesiumbased supports, Catal. Letters. 141 (2011) 1752-1760.

[15] T.S. Hansen, I. Sádaba, E.J. García-Suárez, A. Riisager, Cu catalyzed oxidation of 5hydroxymethylfurfural to 2,5-diformylfuran and 2,5-furandicarboxylic acid under benign reaction conditions, Appl. Catal. A Gen. 456 (2013) 44-50.

[16] C. Boucher-Jacobs, K.M. Nicholas, Catalytic deoxydehydration of glycols with alcohol reductants, ChemSusChem. 6 (2013) 597-599.

[17] C. Boucher-Jacobs, K.M. Nicholas, Oxo-Rhenium-Catalyzed Deoxydehydration of Polyols with Hydroaromatic Reductants, Organometallics. 34 (2015) 1985-1990.

[18] C.E. Hoyle, C.N. Bowman, Thiol-ene click chemistry., Angew. Chem. Int. Ed. Engl. 49 (2010) 1540-1573.

[19] A.B. Lowe, Thiol-ene "click" reactions and recent applications in polymer and materials synthesis, Polym. Chem. 1 (2010) 17.

[20] J.-P. Fouassier, J.F. Rabek, Radiation curing in polymer science and technology, 1st ed., Springer Netherlands, 1993.

[21] C.E. Hoyle, T.Y. Lee, T. Roper, Thiol-enes: Chemistry of the past with promise for the future, J. Polym. Sci. Part A Polym. Chem. 42 (2004) 5301-5338.

[22] C.F. Carlborg, T. Haraldsson, K. Öberg, M. Malkoch, W. van der Wijngaart, Beyond PDMS: off-stoichiometry thiol-ene (OSTE) based soft lithography for rapid prototyping of microfluidic devices, Lab Chip. (2011) 3136-3147.

[23] C. Hoffmann, M. Pinelo, J.M. Woodley, A.E. Daugaard, Development of a thiol-ene based screening platform for enzyme immobilization demonstrated using horseradish peroxidase, Biotechnol. Prog. 33 (2017) 1267-1277.

[24] J.P. Lafleur, S. Senkbeil, J. Novotny, G. Nys, N. Bøgelund, K.D. Rand, F. Foret, J.P. Kutter, Rapid and simple preparation of thiol-ene emulsion-templated monoliths and their 
application as enzymatic microreactors., Lab Chip. 15 (2015) 2162-2172.

[25] P. Mazurek, A.E. Daugaard, M. Skolimowski, S. Hvilsted, A.L. Skov, RSC Advances Preparing mono-dispersed liquid core PDMS microcapsules from thiol - ene - epoxy-tailored, RSC Adv. 5 (2015) 15379-15386.

[26] O.Z. Durham, H.R. Norton, D.A. Shipp, Functional polymer particles via thiol-ene and thiolyne suspension "click" polymerization, RSC Adv. 5 (2015) 66757-66766.

[27] R. Andrisano, Ultraviolet spectra of some furandicarboxylic derivatives, Gazz. Chim. Ital. 81 (1951) 414-418.

[28] B. Ndaba, I. Chiyanzu, S. Marx, N-Butanol derived from biochemical and chemical routes: A review, Biotechnol. Reports. 8 (2015) 1-9.

[29] R. Wölfel, N. Taccardi, A. Bösmann, P. Wasserscheid, Selective catalytic conversion of biobased carbohydrates to formic acid using molecular oxygen, Green Chem. 13 (2011) 2759-2763.

[30] A. Morschbacker, Bio-Ethanol Based Ethylene, Polym. Rev. 49 (2009) 79-84.

[31] J.J. Su, Y.C. Chang, Y.J. Chen, K.C. Chang, S.Y. Lee, Hydrogen sulfide removal from livestock biogas by a farm-scale bio-filter desulfurization system, Water Sci. Technol. 67 (2013) 12881293.

[32] O. Novotný, K. Cejpek, J. Velíšek, Formation of carboxylic acids during degradation of monosaccharides, Czech J. Food Sci. 26 (2008) 117-131. 
Highlights

1. Green, sustainable synthesis of new FDCA and allyl alcohol based building block.

2. Bio-based films from UV-crosslinking with multifunctional thiol compounds.

3. Thorough screening for suitable UV initiator with model system.

4. In-depth real-time FT-IR analyses of reaction kinetics.

5. Evaluation of thermal and mechanical properties of films by DSC, TGA and tensile testing. 
Graphical abstract

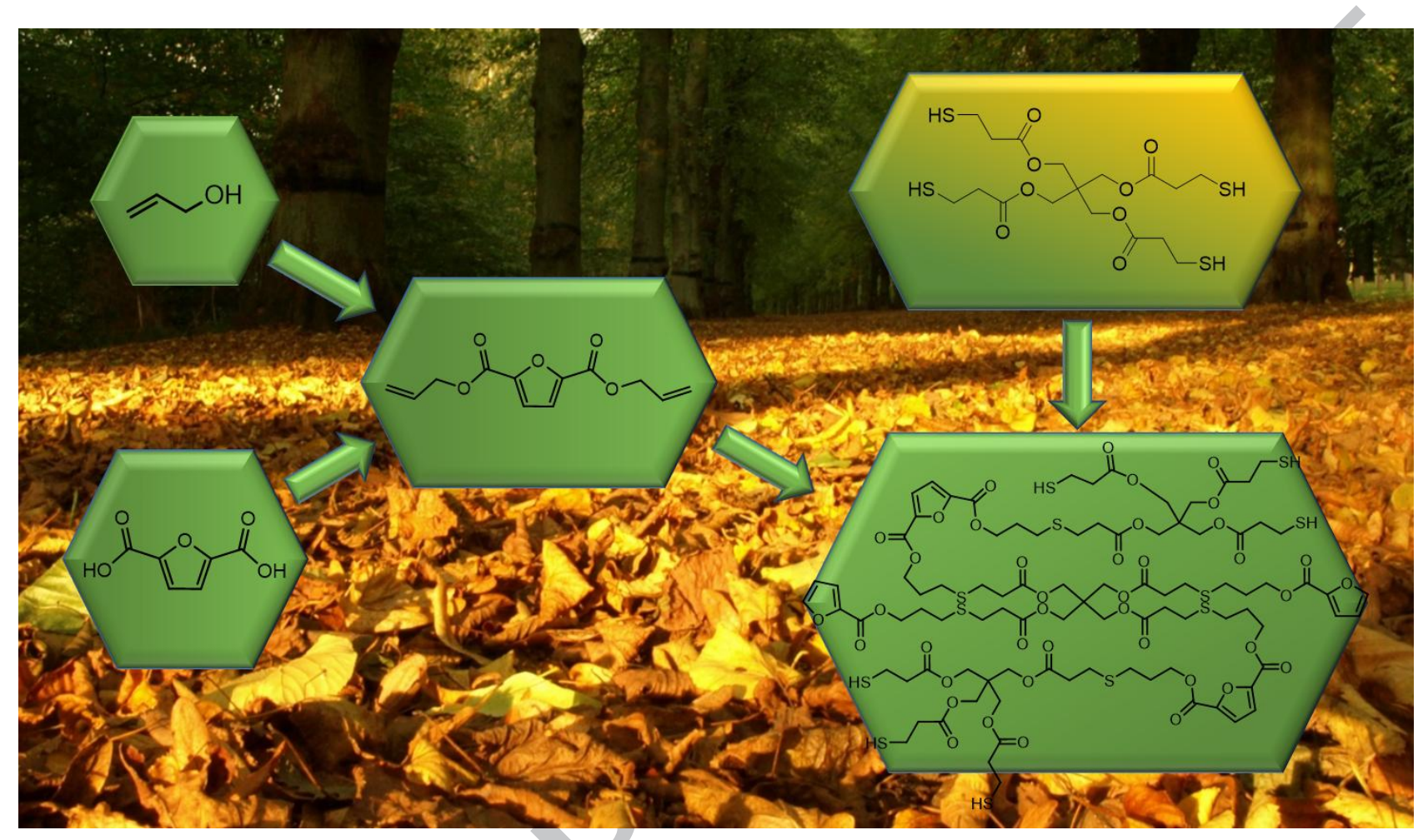

